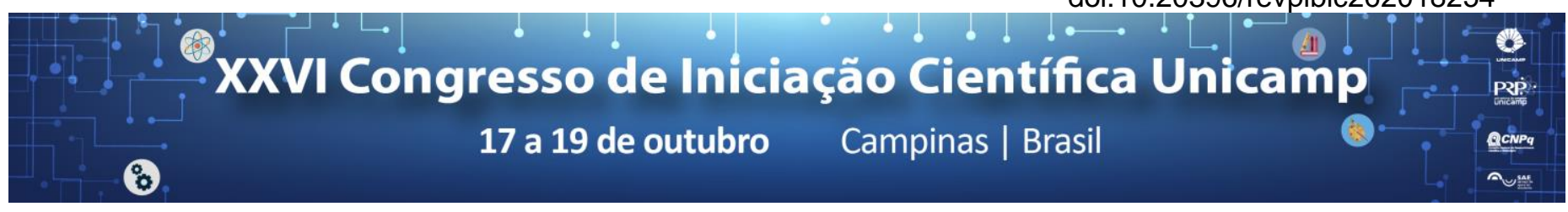

\title{
Investigation of EEG signals generated by motor imagery for application in $\mathrm{BCls}$
}

\author{
Caique M. Mendes*, Carlos A. Stefano Filho, Gabriela Castellano
}

\begin{abstract}
Motor imagery (MI) is a commonly used strategy in brain-computer interfaces (BCls) to modify neuronal activity, in which the user, by imagining motor movements, generates signals that can be recorded and interpreted to control a device. In this study, we sought to investigate how the brain response of users during MI happens, by analyzing a database of EEG signals in which healthy subjects were asked to imagine the movement of their right and left hands. Our goal was to recognize patterns associated with this task, through a spectral evaluation of different segments of the signal. Therefore, we estimated the power spectral density (PSD) for each evaluated segment and then used it for classification, via k-nearest neighbors ( $\mathrm{k}-\mathrm{NN})$. We found that the accuracy rates obtained with k-NN classification were very similar to random, suggesting, mainly, high inter-subjects variability and choice of a low complexity classifier.
\end{abstract}

\section{Key words:}

Electroencephalography, brain-computer interface, motor imagery.

\section{Introduction}

A brain-computer interface $(\mathrm{BCl})$ is a system that measures brain activity in order to translate it into commands that operate an application. Electroencephalography (EEG) has been the most used technique in $\mathrm{BCl}$ systems to record brain activity. One of the strategies to generate the signals captured by EEG is motor imagery (MI), which may be seen as the mental rehearsal of a motor task without its execution, allowing, in principle, the control of a $\mathrm{BCl}$ device ${ }^{1}$. In this study, we sought to investigate how the brain response of users during $\mathrm{MI}$ happens, by analyzing a database of EEG signals in which healthy subjects were asked to imagine the movement of their hands. Our goal was to attempt to recognize patterns associated with this task, through a spectral evaluation of different segments of the signal.

\section{Results and Discussion}

Sixty-four channel EEG data of eight healthy subjects (7 men, age $24 \pm 4$ years) were provided by a previous database collected by our group. The project was approved by the local ethics committee and all subjects gave their written consent. The datasets consisted of MIbased acquisitions for right and left hands, with blocks of rest in between. A single task or rest block lasted $10 \mathrm{~s}$. A standard preprocessing procedure was used for the signals: bandpass filtering $(0.5$ to $50 \mathrm{~Hz})$, identification and removal of bad channels (channels with a low recording signal-to-noise ratio), exclusion of artifactual portions of the data, and common average reference (CAR) filter. Estimates of power spectral density (PSD) of the signal were calculated per second for $\mu$ and $\beta$ bands ( 8 to $30 \mathrm{~Hz}$ ), singly and combined, and then used as features for classification. The classifier used was knearest neighbors (k-NN), given the simplicity of its implementation. Since we aimed to analyze different segments of the signal, we compared the features from the first second of each block (PSD1s) with the first two seconds (PSD2s) and the features from the entire block (PSD10s). Signal from electrodes C1, C2, C3, C4 and Cz were considered so far, owing to their proximity to sensorimotor regions of the brain.
Image 1 shows the performance of the k-NN classifier, in terms of average accuracy, for $\mu$ and $\beta$ bands combined. "Group A" indicates classification among "rest", "right hand MI" and "left hand MI" possibilities; while "Group B" indicates classification between "rest" and "MI" (i.e., right hand plus left hand $\mathrm{MI}$ ) possibilities. Other frequency bands analyses showed similar results. As demonstrated, the accuracy rates obtained with k-NN classifier are very similar to random - that would be $33 \%$ for "Group A" and $50 \%$ for "Group B" - regardless of the feature used.

Image 1. Performance of the classifier: $\mu$ and $\beta$ bands

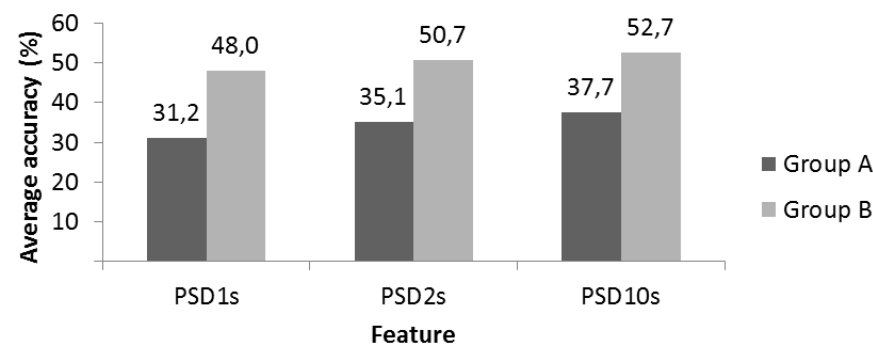

\section{Conclusions}

We believe that these findings resulted, mainly, from: (1) inter-subjects variability, since we used the samples from all the subjects together, in order to have enough samples to study the different segments of the signal; (2) choice of a low complexity classifier for the analysis of high complexity data; (3) data use from few electrodes; (4) difficulty of one's brain to comprehend what would be a "motor rest", inherent to the MI paradigm.

\section{Acknowledgement}

This work was supported by FAPESP (Fundação de Amparo à Pesquisa do Estado de São Paulo) and FINEP (Financiadora de Estudos e Projetos).

\footnotetext{
1 PFURTSCHELlER, G.; NEUPER, C. Motor imagery and direct braincomputer communication. Proceedings of the IEEE, v. 89, n. 7, p. 1123-1134 2001.
} 